\title{
The Evolution of Evolutionary Computation
}

\author{
Xin Yao \\ School of Computer Science at the University of Birmingham, UK \\ $\mathrm{x} \cdot$ yao@cs.bham.ac.uk
}

Evolutionary computation has enjoyed an incredible growth in recent years. This talk will highlight a few recent examples in evolutionary computation in terms of its applications, including data-driven modelling using the evolutionary approach in materials engineering, dynamic route optimisation for salting trucks, multiobjective design of hardware and software, neural network ensemble learning for pattern classification, and online ensemble learning in the presence of concept drifts. The primary objective of this talk is to illustrate novel applications of various evolutionary computation techniques, rather than to go into depth on any of the examples. However, I would be delighted to go into the depth on any of the topics if there is an interest. 